\title{
DNA Knotting Caused By Head-on Collision Of Transcription And Replication
}

Running title: DNA Knotting, Transcription and Replication

Keywords: DNA replication / Transcription / 2D agarose gel electrophoresis / Knotting /

Supercoiling / Replication Fork Barriers (RFBs)

Olavarrieta, L.; P. Hernández; D.B. Krimer and J.B. Schvartzman

Departamento de Biología Celular y del Desarrollo,

Centro de Investigaciones Biológicas (CSIC), Velázquez 144, 28006 Madrid, SPAIN.

* Corresponding author:

Jorge B. Schvartzman

Departamento de Biología Celular y del Desarrollo Centro de Investigaciones Biológicas (CSIC)

Velázquez 144, 28006 Madrid, SPAIN

Phone: ( 34 ) 91 564-4562 ext. 4233

( 34 ) 91 561-1800 ext. 4233

FAX: $\quad$ ( 34 ) $91564-8749$

E-mail: schvartzman@cib.csic.es 


\section{ABSTRACT}

Collision of transcription and replication is uncommon, but the reason for nature to avoid this type of collision is still poorly understood. In Escherichia coli pBR322 is unstable and rapidly lost without selective pressure. Stability can be rescued if transcription of the tetracycline-resistance gene $\left(T e t^{R}\right)$, progressing against replication, is avoided. We investigated the topological consequences of the collision of transcription and replication in pBR322-derived plasmids where head-on collision between the replication fork and the RNA polymerase transcribing the $T e t^{R}$ gene was allowed or avoided. The results obtained indicate that this type of collision triggers knotting of the daughter duplexes behind the fork. We propose this deleterious topological consequence could explain the instability of pBR322 and could be also one of the reasons for nature to avoid head-on collision of transcription and replication. 
Collision of transcription and replication is generally avoided in nature with very few exceptions $(1 ; 2)$. Moreover, in some cases where this is unavoidable, such as in the eukaryotic rDNA loci, a specific mechanism developed to stall the replication fork progressing against transcription just prior to the 3 ' end of the transcriptional unit $(3 ; 4 ; 5)$. The fact that this feature is conserved from bacteria to vertebrates implies it has an unambiguous evolutionary advantage $(6 ; 7)$. But the reason for cells to avoid this type of collision is not fully understood. As in prokaryotes DNA polymerase moves along the DNA template at least $10 \mathrm{x}$ faster than RNA polymerase, the possibility exists for the replication complex to encounter a transcription complex when both are co-oriented as well as when they progress against each other. Both types of collision were studied using the bacteriophages $\mathrm{T} 4$ and $\Phi 29$ in vitro systems $(8 ; 9 ; 10 ; 11)$. The results obtained indicate that the replication fork pauses when it meets a head-on RNA polymerase. Experimental evidence that this is true also in vivo was obtained for the transfer RNA (tRNA) genes in Saccharomyces cerevisiae (2). But the pause caused by the physical contact of an RNA polymerase with the proteins at a replication fork may not be the primary cause for evolution to avoid head-on collision of transcription and replication (1). The DNA template accumulates $(+) \Delta \mathrm{Lk}$ ahead of an actively transcribing gene (12). Unwinding of the helix by DNA helicase during replication also 
leads to positive superhelical turns in the unreplicated template ahead of the fork $(13 ; 14 ; 15)$. Bacterial DNA gyrase introduces $(-) \Delta \mathrm{Lk}$ in this region but this is not enough to compensate all the $(+) \Delta \mathrm{Lk}$ that builds up ahead of the fork, at least during replication. Champoux and Bean (16) suggested that the $(+) \Delta \mathrm{Lk}$ generated ahead of the fork during replication distributes both ahead of and behind the replication fork. To distinguish between $\Delta \mathrm{Lk}$ in the unreplicated portion and in the replicated one, Peter and co-workers (14) call "supercoils" to the first and "precatenanes" to the latter. It is now accepted that during DNA replication in bacteria, topo IV (removing precatenanes in the replicated region) helps DNA gyrase (introducing negative supercoils in the unreplicated region) to eliminate all the (+) $\Delta \mathrm{Lk}$ that builds up during replication $(13 ; 14 ; 15 ; 17)$. The net $\Delta \mathrm{Lk}$ of a plasmid at any time results from the balance between the activities of these three enzymes.

pBR322 is not a natural E. coli plasmid. Bolívar and co-workers (18) constructed this plasmid as a multipurpose cloning system. It has a unidirectional ColE1 replication origin and codes for two antibiotic resistance genes: ampicillin $\left(A m p^{R}\right)$ and tetracycline $\left(T e t^{R}\right)$. The $A m p^{R}$ gene is co-oriented with the ColE1 origin but the $T e t^{R}$ gene is inversely oriented (Figure 1). As the $T_{e}^{R}$ gene transcribes constitutively, collision with the 
replication fork is unavoidable during replication. Moreover, in bacteria grown in the presence of ampicillin, the simultaneous transcription of $A m p^{R}$ and $T e t^{R}$ genes in non-replicating plasmids leads to the formation of twin supercoiling domains where $(+) \Delta \mathrm{Lk}$ accumulates in the intergenic region (19). pBR322 DNA showing net (+) $\Delta \mathrm{Lk}$ was clearly identified in chloroquine 2D gels after inhibition of DNA gyrase with novobiocin and this positive supercoiling depends on the presence and orientation of the Tet ${ }^{R}$ gene (19). pBR322 knotted forms was observed in E. coli topoisomerase mutants and most of the nodes of these knots have a negative sign (20). Formation of these knotted plasmids also depends on the presence and orientation of the $T e t^{R}$ gene (21). In agreement with these observations, pBR322 DNA isolated from DH5 $\alpha \mathrm{F}$ ' cells, carrying no topoisomerase mutations, reveals significant amounts of knotted plasmids as well as molecules showing low levels of supercoiling when analyzed by the Brewer-Fangman neutral/neutral (N/N) two-dimensional (2D) agarose gel electrophoresis (22). Altogether, these observations indicate that in pBR322, transcription of the $T e t^{R}$ gene is responsible for the particular topological characteristics of this plasmid in E. coli cells $(19 ; 20 ; 21)$. Stability of pBR322-derived plasmids during growth of their E. coli host in the absence of antibiotics has been studied in detail. pBR322 was found to be very unstable under these conditions and was lost within $\sim 60$ 
generations. But a number of derivatives where the promoter of the $T e t^{R}$ gene had been deleted, were stable under the same conditions (23). This observation indicates that transcription of the $T e t^{R}$ gene is responsible also for the instability of pBR322 in the absence of selective pressure.

It was recently shown that ColE1 plasmids containing a stalled fork could be knotted. But these knots occur in the replicated region behind the fork (24). Most of the nodes of these knotted bubbles have a positive sign (25) indicating that they resulted from in vivo action of a type II topoisomerase on negatively twisted precatenanes (26).

$\mathrm{N} / \mathrm{N} 2 \mathrm{D}$ agarose gel electrophoresis was originally designed to separate branched from linear molecules (27), but this technique can be used also to resolve the different forms undigested circular DNA can adopt $(22 ; 28 ; 29 ; 30)$. In the present report we used this technique to compare the patterns observed for several pBR322-derived plasmids where transcription of the $T e t^{R}$ gene was on or off and where progression of the DNA replication fork was blocked either before or after the $T e t^{R}$ gene.

pBR18-TerE@StyI and pBR322-TerE@StyI were constructed inserting the $23 \mathrm{bp}$ that constitutes the E. coli polar replication terminator 
TerE $(31 ; 32)$ in its active orientation between the unique StyI and AvaI sites of pBR18 or pBR322 (Figure 1). pBR18 is a derivative of pBR322 where the nucleotides between the unique EcoRI and HindIII sites had been replaced with the polycloning fragment of pUC18 (33). In doing so, the promoter for the $T e t^{R}$ gene is lost. Thus, the main difference between pBR18 and pBR322 is that pBR18 lacks the promoter for the $T e t^{R}$ gene (Figure 1). We anticipated that in both plasmids, replication forks would stop at the TerE-TUS complex leading to the accumulation of specific RIs containing an internal bubble and with a total mass $1.26 \mathrm{x}$ the mass of nonreplicating plasmids. It was previously shown that in order to reveal the presence of knotted bubbles, $\Delta \mathrm{Lk}$ has to be eliminated $(24 ; 25 ; 33 ; 34) . E$. coli DH5 $\alpha \mathrm{F}^{\prime}$ cells were transformed with either pBR18-TerE@StyI or pBR322-TerE@StyI and plasmid DNAs were digested with ScaI, a restriction enzyme that cuts both plasmids only once and outside the putative replicated region. Then linearized molecules were analyzed by $\mathrm{N} / \mathrm{N} 2 \mathrm{D}$ agarose gel electrophoresis (35). Autoradiograms of these gels are shown in Figure 2 with corresponding diagrammatic interpretations to their right. These autoradiograms revealed that in both cases the vast majority of partially replicated plasmids had a fork stalled at TerE. In other words, after digestion with ScaI, pBR18-TerE@StyI and pBR322-TerE@StyI occurred in only two basic forms: the non-replicating (1.0x) linear form 
and the partially replicated RI containing an internal bubble (1.26xBubble). To the right of the accumulated 1.26xBubble, a rather short "beads-on-astring" signal was observed. This signal corresponded to knotted bubbles $(24 ; 25 ; 33 ; 34)$. No significant difference in the number and complexity of knotted bubbles was observed in the autoradiograms corresponding to pBR18-TerE@StyI and pBR322-TerE@StyI.

pBR18-TerE@AatII and pBR322-TerE@AatII were constructed inserting TerE $(31 ; 32)$ at the unique AatII site of pBR18 or pBR322 (Figure 1). 2D gel autoradiograms of these plasmids after restriction digestion with $A l w \mathrm{NI}$ are shown in Figures $3 \mathrm{~A}$ and $\mathrm{B}$, with diagrammatic interpretations to their right. Signals detected below the accumulated bubbles were likely due to single-stranded breakage of replication intermediates (RIs) containing an internal bubble and trailing during the first dimension due to overloading was responsible for the tails detected for the most abundant molecular species in the autoradiograms (36). A densitometric analysis of the "beads-on-a-string" signal corresponding to unknotted and knotted bubbles is included above each autoradiogram. The most significant difference between these two plasmids was the number and complexity of knotted bubbles. The densitometric profiles confirmed this observation. When the strength of the signal corresponding to 
unknotted bubbles of both profiles was made equal, there were $64 \%$ more knotted bubbles in pBR322-TerE@AatII.

The excess of knotted bubbles observed for pBR322-TerE@AatII could be due to the head-on collision of transcription and replication. But the possibility existed also that this extra knotting could be caused by transcription of the $T e t^{R}$ gene itself regardless of whether it occurred against or co-oriented with replication fork progression. To test this latter possibility, a new plasmid was made where the EcoRI-StyI restriction fragment of pBR322-TerE@AatII was inverted (see Figure 1). In the new plasmid (pBR322-TerE@AatII-inv) transcription of the $T e t^{R}$ gene was cooriented with progression of the replication fork. The corresponding 2D gel autoradiogram after restriction digestion with $A l w \mathrm{NI}$ is shown in Figure $3 \mathrm{C}$, with a diagrammatic interpretation to its right. Note that in the new plasmid the number of knotted bubbles was even lower than for pBR18TerE@AatII (Figure 3A), where the promoter of the $T e t^{R}$ gene had been deleted. This isn't unexpected, though, as in pBR18-TerE@AatII some transcription could still take place, although at a very low rate, driven from cryptic promoters located upstream the deleted one (36). Note that this putative low level of transcription, however, was not sufficient to turn the cells resistant to tetracycline, probably because these transcripts were not 
translated properly. The observation that pBR322-TerE@AatII-inv exhibited the lowest number of knotted bubbles strengthens the idea that the excess of knotted bubbles observed for pBR322-TerE@AatII was indeed caused by head-on collision of transcription and replication.

Knotted bubbles were originally detected in ColE1 plasmids where replication forks pause or are permanently blocked at a Ter site or at another ColE1 origin with the opposite orientation $(24 ; 25 ; 33 ; 34)$. This type of knots reflects the number and pattern of DNA crossings trapped between the two segments participating in the strand passage event (25; 26). As knotted bubbles occur in the replicated portion of partially replicated plasmids, the two segments involved are the two daughter duplexes. In those cases where replication and transcription progress against each other, $(+) \Delta \mathrm{Lk}$ accumulates in the region between the two advancing forks. This change in topology rapidly diffuses to the replicated portion where it changes the number of precatenanes facilitating DNA knotting. Blockage of the replication fork at TerE with the concomitant formation of some knotted bubbles occurred in all the plasmids we have studied alike. But the increased number of knotted bubbles observed for pBR322-TerE@AatII was due to some extra-knotting that took place only 
in this plasmid where the replication fork progressed against the RNA polymerase transcribing the $T e t^{R}$ gene (Figure 4B).

Why most of the nodes of non-replicating knotted plasmids of pBR322 have a negative sign (20) while the sign of the nodes of knotted bubbles are predominantly positive (25)? Knotted bubbles form when a type II topoisomerase crosses two successive precatenanes (26). For this reason positive supercoiling leads to DNA knots having predominantly negative nodes while negative supercoiling leads to knots with positive nodes $(37 ; 38)$. In pBR322 the opposing orientation of $A m p^{R}$ and $T e t^{R}$ genes leads to the accumulation of (+) $\Delta \mathrm{Lk}$ in the intergenic region (19). The nodes of the DNA knots formed in these non-replicating plasmids have a negative sign (20) because the template was positively supercoiled (Figure 4A). On the other hand, due to the combined action of DNA gyrase and topo IV, partially replicated ColE1 plasmids display negatively twisted precatenanes (Figure 4B). For this reason the sign of the nodes of knotted bubbles are predominantly positive (25).

The biological significance of knotted bubbles is still unknown, but DNA knotting has potentially devastating effects on cells (39). It was 
recently shown that topo IV alone is responsible for unknotting DNA in $E$. coli cells (40). It is conceivable that topo IV should be able to eliminate most knotted bubbles in vivo, but too many knots are likely to delay or severely interfere with normal segregation. The excess of knotted bubbles observed for pBR322-TerE@AatII could explain the instability of pBR322 in E. coli cells in the absence of selective pressure (23). Altogether, these observations led us to propose the deleterious consequence of this excess of DNA knotting, and not just the physical collision of an RNA polymerase with the proteins at a replication fork, as the main reason for nature to avoid head-on collision of transcription and replication.

\section{ACKNOWLEDGEMENTS}

We are grateful to Andrzej Stasiak for his advices, constant stimulation and for leading us into the world of DNA topology, to José Manuel Sogo and Alicia Sánchez-Gorostiaga for their support throughout the course of this study and to Marisa Martínez-Robles and Pilar Robles for technical assistance. This work was partially supported by grants PM970138 and PGC PB98-048 from the Spanish Comisión Interministerial de Ciencia y Tecnología (CICYT), 99/0850 from the Spanish Fondo de 
Investigación Sanitaria (FIS) and 08.6/0016/1997 from the Comunidad Autónoma de Madrid (CAM).

\section{REFERENCES}

1. Brewer, B. J. (1988). When polymerases collide: replication and the transcriptional organization of the E coli chromosome. Cell 53, 679686.

2. Deshpande, A. M. \& Newlon, C. S. (1996). DNA replication fork pause sites dependent on transcription. Science 272, 1030-1033.

3. Linskens, M. H. K. \& Huberman, J. A. (1988). Organization of replication of ribosomal DNA in Saccharomyces cerevisiae. Mol Cell Biol 8, 4927-4935.

4. Brewer, B. J. \& Fangman, W. L. (1988). A replication fork barrier at the 3' end of yeast ribosomal RNA genes. Cell 55, 637-643.

5. French, S. (1992). Consequences of replication fork movement through transcription units in vivo. Science 258, 1362-1365.

6. Hernández, P., Martín-Parras, L., Martínez-Robles, M. L. \& Schvartzman, J. B. (1993). Conserved features in the mode of 
replication of eukaryotic ribosomal RNA genes. EMBO J. 12, 14751485.

7. López-Estraño, C., Schvartzman, J. B., Krimer, D. B. \& Hernández, P. (1998). Co-localization of polar replication fork barriers and rRNA transcription terminators in mouse rDNA. J Mol Biol 277, 249-256.

8. Liu, B., Wong, M. L., Tinker, R. L., Geiduschek, E. P. \& Alberts, B. M. (1993). The DNA replication fork can pass RNA polymerase without displacing the nascent transcript. Nature 366, 33-39.

9. Liu, B. \& Alberts, B. M. (1995). Head-on collision between a DNA replication apparatus and RNA polymerase transcription complex. Science 267, 1131-1137.

10. Elias-Arnanz, M. \& Salas, M. (1997). Bacteriophage phi 29 DNA replication arrest caused by codirectional collisions with the transcription machinery. EMBO J 16, 5775-5783.

11. Elias-Arnanz, M. \& Salas, M. (1999). Resolution of head-on collisions between the transcription machinery and bacteriophage Phi 29 DNA polymerase is dependent on RNA polymerase translocation. EMBO J $18,5675-5682$.

12. Liu, L. F. \& Wang, J. C. (1987). Supercoiling of the DNA template during transcription. Proc Natl Acad Sci USA 84, 7024-7027. 
13. Alexandrov, A. I., Cozzarelli, N. R., Holmes, V. F., Khodursky, A. B., Peter, B. J., Postow, L., Rybenkov, V. \& Vologodskii, A. V. (1999). Mechanisms of separation of the complementary strands of DNA during replication. Genetica 106, 131-140.

14. Peter, B. J., Ullsperger, C., Hiasa, H., Marians, K. J. \& Cozzarelli, N. R. (1998). The structure of supercoiled intermediates in DNA replication. Cell 94, 819-827.

15. Ullsperger, C., Vologodskii, A. A. \& Cozzarelli, N. R. (1995). Unlinking of DNA by topoisomerases during DNA replication. In Nucleic Acids and Molecular Biology (Lilley, D. M. J. \& Eckstein, F., eds.), pp. 115-142. Springer-Verlag, Berlin.

16. Champoux, J. J. \& Been, M. D. (1980). Topoisomerases and the swivel problem. In Mechanistic studies of DNA replication and genetic recombination (Alberts, B., ed.), pp. 809-815. Academic Press, New York.

17. Zechiedrich, E. L. \& Cozzarelli, N. R. (1995). Roles of topoisomerase IV and DNA gyrase in DNA unlinking during replication in Escherichia coli. Genes Dev 9, 2859-69.

18. Bolívar, F., Rodríguez, R. L., Greene, P. J., Betlach, M. C., Heyneker, H. L., Boyer, H. W., Crosa, J. H. \& Falkow, S. (1977). Construction 
and characterization of new cloning vehicles, II. A multipurpose cloning system. Gene 2, 95-113.

19. Wu, H. Y., Shyy, S., Wang, J. C. \& Liu, L. F. (1988). Transcription generates positively and negatively supercoiled domains in the template. Cell 53, 433-440.

20. Shishido, K., Komiyama, M. \& Ikawa, S. (1987). Increased production of a knotted form of plasmid pBR322 DNA in Escherichia coli DNA topoisomerase mutants. J Mol Biol 195, 215-218.

21. Shishido, K., Ishii, S. \& Komiyaba, N. (1989). The presence of the region on pBR322 that encodes resistance to tetracycline is responsible for high levels of plasmid DNA knotting in Escherichia coli DNA topoisomerase I deletion mutant. Nucleic Acids Res 17, 9749-9759.

22. Martín-Parras, L., Lucas, I., Martínez-Robles, M. L., Hernández, P., Krimer, D. B., Hyrien, O. \& Schvartzman, J. B. (1998). Topological complexity of different populations of pBR322 as visualized by twodimensional agarose gel electrophoresis. Nucleic Acids Res 26, 34243432.

23. Chiang, C. S. \& Bremer, H. (1988). Stability of pBR322-derived plasmids. Plasmid 20, 207-220.

24. Viguera, E., Hernández, P., Krimer, D. B., Boistov, A. S., Lurz, R., Alonso, J. C. \& Schvartzman, J. B. (1996). The ColE1 unidirectional 
origin acts as a polar replication fork pausing site. $J$ Biol Chem 271, 22414-21.

25. Sogo, J. M., Stasiak, A., Martínez-Robles, M. L., Krimer, D. B., Hernández, P. \& Schvartzman, J. B. (1999). Formation of knots in partially replicated DNA molecules. J Mol Biol 286, 637-643.

26. Postow, L., Peter, B. J. \& Cozzarelli, N. B. (1999). Knot what we thought before: the twisted story of replication. Bioessays 21, 805-808.

27. Bell, L. \& Byers, B. (1983). Separation of branched from linear DNA by two-dimensional gel electrophoresis. Anal Biochem 130, 527-535.

28. Brewer, B. J., Sena, E. P. \& Fangman, W. L. (1988). Analysis of replication intermediates by two-dimensional agarose gel electrophoresis. Cancer Cells 6, 229-234.

29. Lucas, I., Germe, T., Chevrier-Miller, M. \& Hyrien, O. (2001). Topoisomerase II can unlink replicating DNA by precatenane removal. EMBO J 20, 6509-6519. 
30. Hanai, R. \& Roca, J. (1999). Two-dimensional agarose-gel electrophoresis of DNA topoisomers. In DNA Topoisomerase Protocols (Bjornsti, M. A. \& Osheroff, N., eds.), Vol. 94, pp. 19-27. Humana Press Inc, 999 Riverview Dr, Ste 208, Totowa, NJ 07512-1165, USA.

31. Hill, T. M., Pelletier, A. J., Tecklenburg, M. L. \& Kuempel, P. L. (1988). Identification of the DNA sequence from E coli terminus region that halts replication forks. Cell 55, 459-466.

32. Bastia, D. \& Mohanty, B. K. (1996). Mechanisms for completing DNA replication. In DNA replication in eukaryotic cells (DePamphilis, M. L., ed.), pp. 177-215. Cold Spring Harbor Laboratory Press, New York.

33. Santamaría, D., Hernández, P., Martínez-Robles, M. L., Krimer, D. B. \& Schvartzman, J. B. (2000). Premature termination of DNA replication in plasmids carrying two inversely oriented ColE1 origins. $J$ Mol Biol 300, 75-82.

34. Olavarrieta, L., Martínez-Robles, M. L., Sogo, J. M., Stasiak, A., Hernández, P., Krimer, D. B. \& Schvartzman, J. B. (2002). Supercoiling, knotting and replication fork reversal in partially replicated plasmids. Nucleic Acids Res 30, 656-666.

35. Brewer, B. J. \& Fangman, W. L. (1987). The localization of replication origins on ARS plasmids in S. cerevisiae. Cell 51, 463-471. 
36. Santamaría, D., delaCueva, G., Martínez-Robles, M. L., Krimer, D. B., Hernández, P. \& Schvartzman, J. B. (1998). DnaB helicase is unable to dissociate RNA-DNA hybrids - Its implication in the polar pausing of replication forks at ColE1 origins. J Biol Chem 273, 33386-33396.

37. Wasserman, S. A. \& Cozzarelli, N. R. (1991). Supercoiled DNAdirected knotting by T4 topoisomerase. J Biol Chem 266, 20567-20573.

38. Krasnow, M. A., Stasiak, A., Spengler, S. J., Dean, F., Koller, T. \& Cozzarelli, N. R. (1983). Determination of the absolute handedness of knots and catenanes of DNA. Nature 304, 559-560.

39. Pieranski, P., Kasas, S., Dietler, G., Dubochet, J. \& Stasiak, A. (2001). Localization of breakage points in knotted strings. New Journal of Physics 3, 10.1-10.13.

40. Deibler, R. W., Rahmati, S. \& Zechiedrich, E. L. (2001). Topoisomerase IV, alone, unknots DNA in E-coli. Genes Develop 15, $748-761$. 


\section{LEGEND TO FIGURES}

Figure 1: Maps of the plasmids used in this study showing the relative position of their most relevant features: the ColE1 unidirectional origin, the E. coli terminator TerE, the $A m p^{R}, T e t^{R}$ and rop genes and the recognition sites for a number of restriction endonucleases. To construct pBR18TerE@StyI and pBR322-TerE@StyI, two oligos:

5'-CTTGGGGCTTAGTTACAACATACTTTAAC-3' and 5'-CCGAGTTAAAGTATGTTGTAACTAAGCCC-3' containing the 23 bp that constitutes the E. coli TerE terminator with a 3' StyI and a 5' AvaI tails were annealed to each other and inserted between the unique StyI and AvaI sites of pBR18 or pBR322. To construct pBR18-TerE@AatII and pBR322-TerE@AatII, two different oligos:

5'-CGTCGACGGCTTAGTTACAACATACTTTAAGACGT-3' and 5'-CTTAAAGTATGTTGTAACTAAGCCGTCGACGACGT-3' with two AatII tails and one SalI site were annealed to each other and inserted at the unique AatII site of pBR18 or pBR322. Construction of pBR322TerE@AatII-inv was performed inverting the EcoRI-StyI fragment of pBR322-TerE@AatII. Transcription of the Tet ${ }^{R}$ gene in pBR322, pBR322TerE@StyI, pBR322-TerE@AatII and pBR322-TerE@AatII-inv was 
confirmed growing the cells transformed with these plasmids at $37^{\circ} \mathrm{C}$ in $\mathrm{LB}$ medium containing $12.5 \mu \mathrm{g} / \mathrm{ml}$ tetracycline.

Figure 2: Autoradiograms of 2D gels corresponding to pBR18-TerE@StyI (A and B, upper panels) and pBR322-TerE@StyI (C and D, lower panels) after digestion with $S c a$ I. No significant differences were observed between both plasmids. The E. coli strain used was DH5 $\alpha$ F'. Competent cells were transformed with monomeric forms of the plasmids as described $(24 ; 34$; 36). Cells were grown at $37^{\circ} \mathrm{C}$ in $\mathrm{LB}$ medium containing $50 \mathrm{mg} / \mathrm{ml}$ ampicillin. Isolation of plasmid DNA, N/N 2D agarose gel electrophoresis, Southern transfer and hybridization were performed as described elsewhere $(22 ; 24 ; 33 ; 34)$.

Figure 3: Autoradiograms of $2 \mathrm{D}$ gels corresponding to pBR18TerE@AatII (A, upper panel), pBR322-TerE@AatII (B, mid panel) and pBR322-TerE@AatII-inv (C, lower panel) after digestion with AlwNI. Note the increased number and complexity of knotted bubbles in pBR322TerE@AatII (B, mid panel). To help visualization of this difference, a densitometric profile of unknotted and knotted bubbles (made using version 1.61 of NIH Image) is shown above each autoradiogram with the profile corresponding to pBR322-TerE@AatII shaded and superimposed on the 
profiles of the other two plasmids. The E. coli strain used was DH5 $\alpha$ F'. Competent cells were transformed with monomeric forms of the plasmids as described $(24 ; 34 ; 36)$. Cells were grown at $37^{\circ} \mathrm{C}$ in $\mathrm{LB}$ medium containing $50 \mathrm{mg} / \mathrm{ml}$ ampicillin. Isolation of plasmid DNA, N/N 2D agarose gel electrophoresis, Southern transfer and hybridization were performed as described elsewhere $(22 ; 24 ; 33 ; 34)$.

Figure 4: Topological consequences of the opposing orientation of two actively transcribing genes (A) and head-on collision of transcription and replication (B) are schematically presented. Head-on orientation of two actively transcribing genes leads to the formation of $(+) \Delta \mathrm{Lk}$ in the intergenic region. This causes topo IV to knot the template. As the DNA was positively supercoiled, the nodes of these knots have predominantly negative signs (upper right corner). Head-on collision of transcription and replication also leads to the accumulation of $(+) \Delta \mathrm{Lk}$. But this positive supercoiling rapidly diffuses behind the replication fork, changing the twisting degree of the two daughter duplexes. This change in topology facilitates the formation of knotted bubbles. As due to the combined action of DNA gyrase and topo IV precatenanes were negatively twisted, the nodes of these knotted bubbles have predominantly positive signs (lower right corner). 


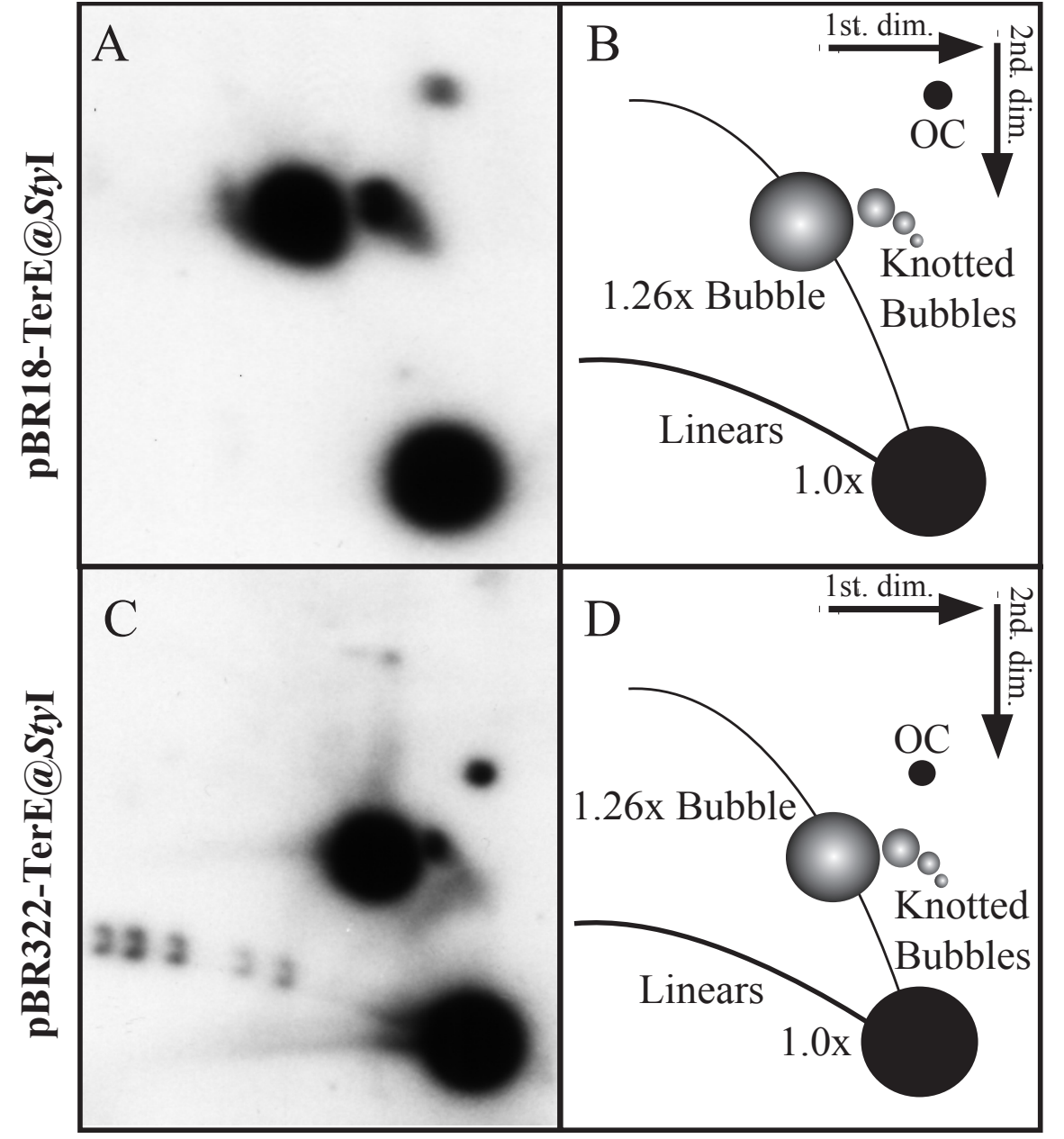




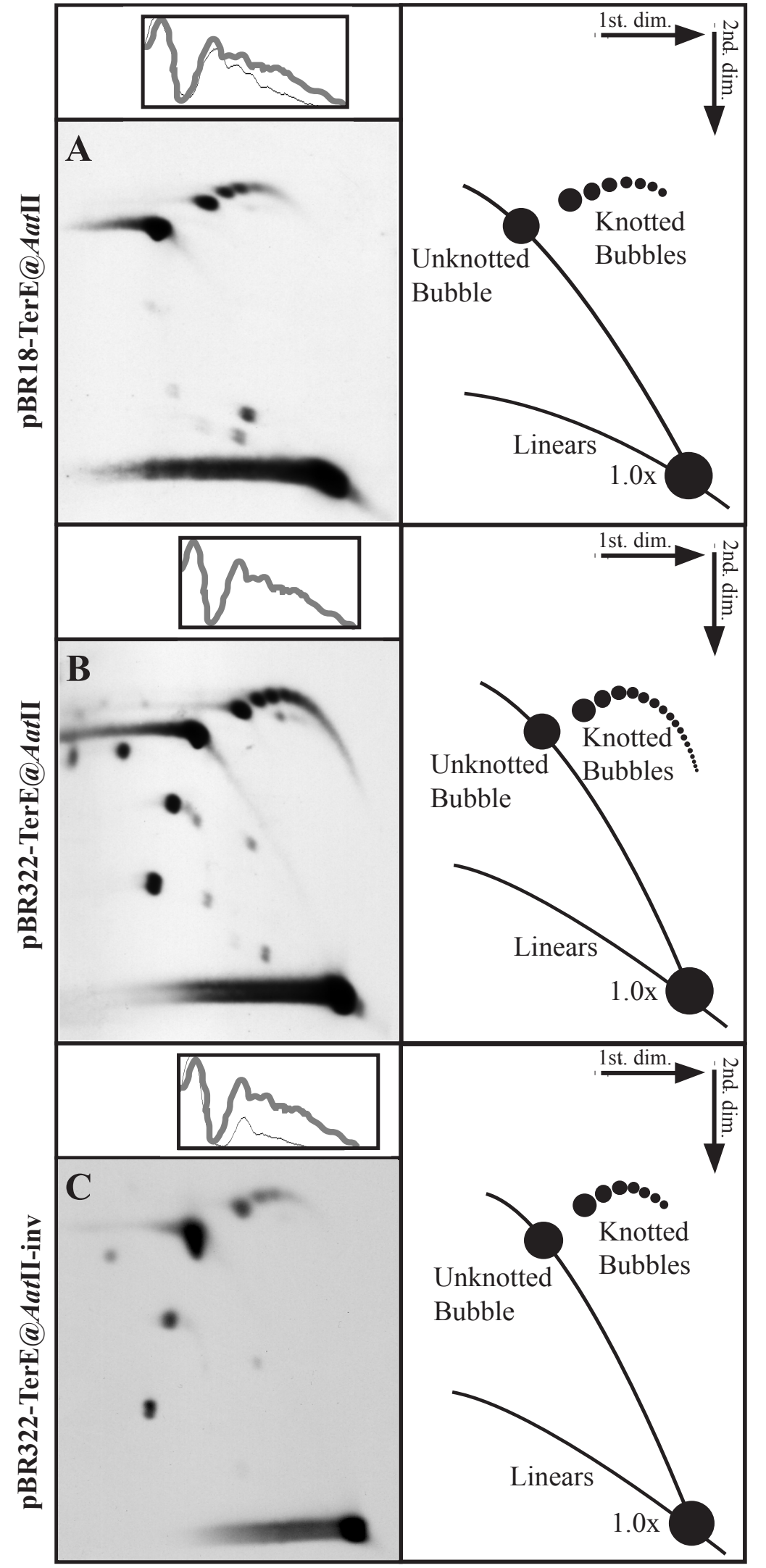




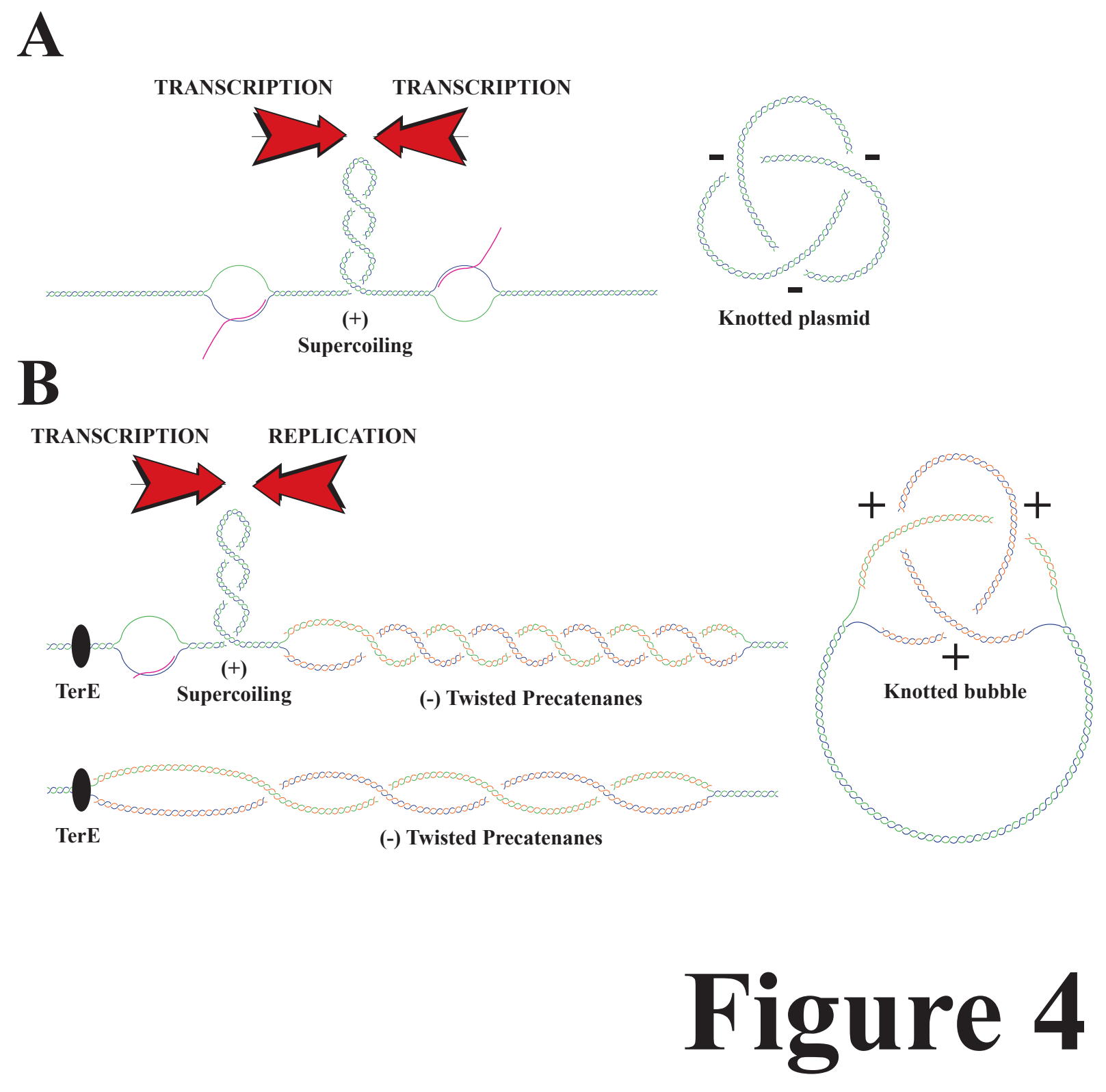

